\title{
ANALYSIS OF GREEN'S FUNCTION AND SURFACE CURRENT DENSITY FOR RECTANGULAR MICROSTRIP PATCH ANTENNA
}

\author{
Pallavi Singhal $^{1}$ \\ ${ }^{I}$ M.Tech Student, Dept. of ECE, KIET, Ghaziabad, U.P., India
}

\begin{abstract}
In this paper, Green's function and surface current density for planar structure has been calculated. The approach makes use of the popular and rigorously used spectral domain full wave analysis method in conjunction with method of moment as numerical analysis tool. In present approach, boundary conditions are applied at patch metallization, which leads to integral equation with the involvement of green's function in spectral domain, which includes the effect of dielectric, conductor loss, surface wave modes and space wave radiation. By applying Galerkin's moment method integral equation are transformed to linear set of equations. Entire domain basis function is used to improve the efficiency of the solution.
\end{abstract}

Keywords: Spectral domain full wave analysis, Green function, Galerkin's moment method, Entire domain basis function, Surface current density.

\section{INTRODUCTION}

In today's modern communication industry, antennas are the most important components required to create a communication link. Through the years, Microstrip patch antenna is the most common option used to realize millimetre wave monolithic integrated circuits for microwave, radar and communication purposes [1]. Several theoretical models, for the analysis of microstrip antenna have been introduced during the past two decades. Among the first two models were the Transmission line model and Cavity model [2]. Both approaches are relatively easy to implement into a computer program and require relatively short computation time. However, with these models the antenna characteristics are not very accurate and are usually limited to the case of narrow band microstrip antennas [3]. Later more rigorous methods have been proposed such as Full Wave Analysis. In this method, the antenna characteristics can be determined by solving the integral equations (method of moment) which involve calculation of closed form Green's functions either in spectral or spatial domain [4]. Although the large volume of literature using numerical methods for the analysis of microstrip patch antenna implicitly involves calculation of Green's function and treating its singularities; yet very little research work has been published explicitly in the domain of Green's function's itself. He Mang and Xu Xiaowen, in 2002, divided the impedance matrix integrand into two parts: one being the smooth part and the other being fast oscillatory and slowly convergent part and dealt with them separately [5]. In 2005, M. I. Aksun and Gülbin Dural presented Clarification of Issues on the Closed-Form Green's Functions in Stratified Media [6]. In 2007, Alexander svezhentsev and guy vandenbosch reported two types of bad behaviour of Green's function in spatial domain [7]. In this paper, spectral- domain full wave approach is used to analyze patch over rectangular surface which involves Green's function. Surface current density is obtained from method of moment application by assuming current variation in $\mathrm{z}$ direction only.

\section{CHARACTERSTICS OF MICROSTRIP PATCH} ANTENNA

In their simple geometry, microstrip Patch antenna consists of a radiating patch on one side of a dielectric substrate which has a ground plane on the other side as shown in fig. 1 . Microstrip patch antennas are increasing in popularity for use in wireless applications due to their low-profile structure. Therefore they are extremely compatible for embedded antennas in handheld wireless devices such as cellular phones, pagers etc. The telemetry and communication antennas on missiles need to be thin and conformal and are often in the form of Microstrip patch antennas. It has been often observed that the microstrip antenna can easily be integrated with a feed network and circuitry on the same substrate. If this is done, some compromise has to be made between good antenna performance and good circuit performance since for good antenna performance thick substrates with low dielectric constant is used that results in larger antenna size. In order to design a compact antenna, high dielectric constant substrate is used which results in poor efficiency and narrow bandwidth [8]. 


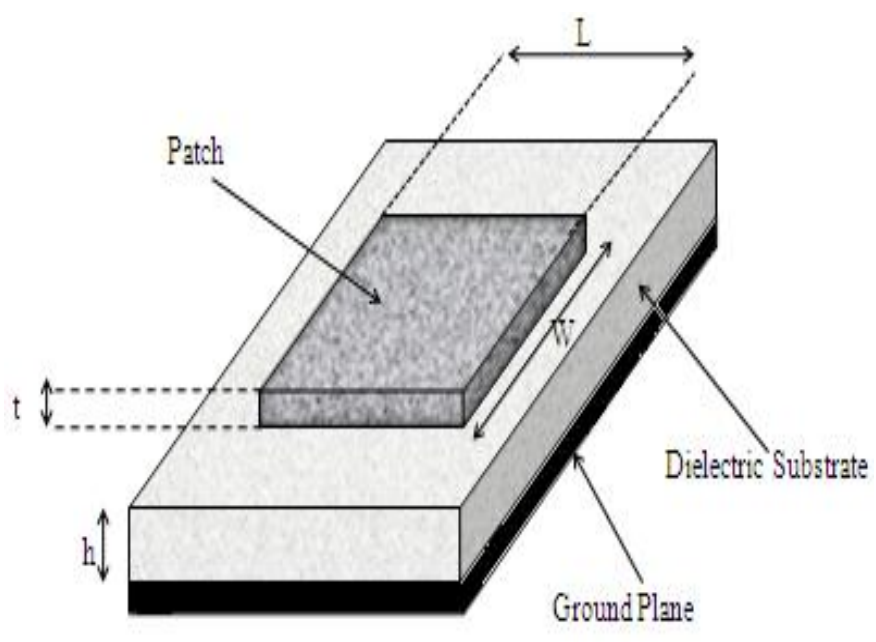

Fig.-1. Basic Geometry of Microstrip Antenna

\section{DESIGN PARAMETERS}

Here we have designed the rectangular microstrip patch antenna for the S-band frequency ranges ( $3 \mathrm{GHz}$ in this case). Microstrip patch antenna working on this frequency range finds application in weather radar, surface ship radar, and some communications satellites. The design flow for the single patch dimensions, using the cavity model approach is as follows:

Fr, frequency of operation $=3 \mathrm{GHz}$

Err, relative permittivity of substrate material $=2.32$

$\mathrm{h}$, height of substrate $=0.159 \mathrm{~cm}$

Calculated length and width of patch are:

$\mathrm{L}$, length of patch $=3.2 \mathrm{~cm}$

$\mathrm{W}$, width of patch $=3.8 \mathrm{~cm}$

The dominant mode is thus TM01. The results have been obtained using MATLAB-2007b.

\subsection{Analysis Approach}

The approach makes use of the popular and rigorously used spectral domain full wave analysis method in conjunction with method of moment as numerical analysis tool. In the analysis, the solution for electric field due to rectangular patch is obtained by solving integral equation which involves Green's function in spectral domain. The Green's function is very strong mathematical tool to solve differential equations subjected to certain boundary conditions [9]. The integral equations thus formed are converted into a system of linear equations by the use of method of moment. In the method of moment, the unknown patch current is expressed into a set of linear combination of basis function weighted by unknown coefficients which are determined by the solution of linear system of equation. In general, two types of basis functions are used; Entire Domain Basis Function and Sub-Domain Basis Function. The present work thus makes use of entire domain basis functions to expand unknown patch current density due to major disadvantage of sub-domain basis function that is, they require more computation time and computer memory than properly chosen entire domain basis function [10]. A rectangular patch of dimensions $L$ and $W$ has been assumed on infinite substrate of height $\mathrm{h}$ and permittivity $\varepsilon$. Below is infinite ground plane.

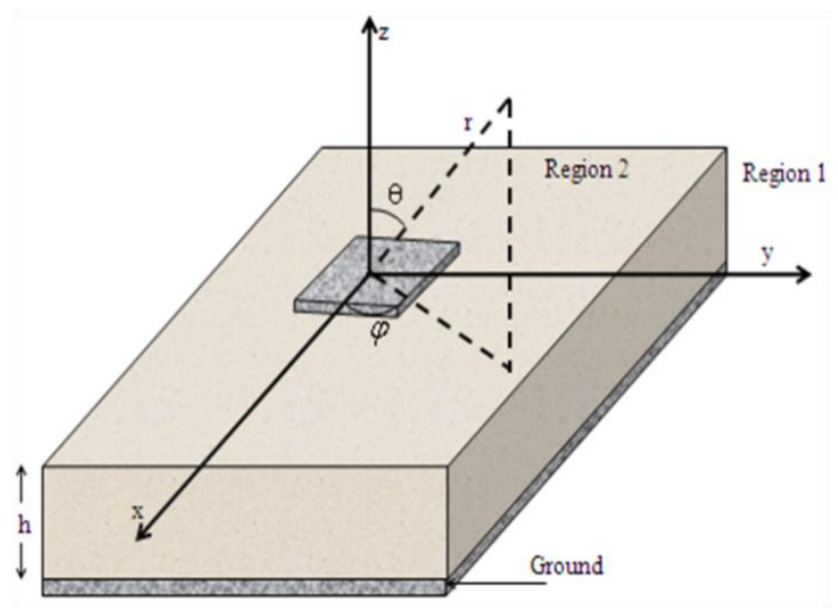

Fig- 2 Rectangular Patch Antenna

To solve for the rectangular patch on given geometry, spectral domain Green's functions have been calculated and then Moment Method approach is applied to solve for coefficients of unknown patch current density.

Electric and magnetic fields in region- 1 and region- 2 are given as:

In region $1,0<\mathrm{z}<\mathrm{h}$,

$\tilde{E}_{z 1}=A_{1} \cos \left(k_{1} z\right)+B_{1} \sin \left(k_{1} z\right)$

$\tilde{H}_{z 1}=C_{1} \sin \left(k_{1} z\right)+D_{1} \cos \left(k_{1} z\right)$

In region $2, \mathrm{z}>\mathrm{h}$,

$\tilde{E}_{z 2}=A_{2} e^{-j k_{2} z}$

$\tilde{H}_{z 2}=C_{2} e^{-j k_{2} z}$

Where $A_{1}, B_{1}, C_{1}, D_{1}, A_{2}, C_{2}$ are unknown coefficients to be found by application of boundary conditions.

Applying boundary conditions on two boundaries; $\mathrm{z}=0$ and $\mathrm{z}=\mathrm{h}$,

$$
\tilde{E}_{x}=0 a t z=0
$$




$$
\tilde{E}_{y}=0 a t z=0
$$

Continuity of $\widetilde{E}_{x}$ at $z=h$ i.e $\widetilde{E}_{x 1}=\widetilde{E}_{x 2}$

Continuity of $\widetilde{E}_{y}$ at $z=h$ i.e $\widetilde{E}_{y 1}=\widetilde{E}_{y 2}$

Continuity of $\tilde{H}_{x}$ at $z=h$ i.e $\tilde{H}_{x 1}=\tilde{H}_{x 2}$

Continuity of $\tilde{H}_{y}$ at $z=h$ i.e $\tilde{H}_{y 1}-\tilde{H}_{y 2}=\widetilde{J}_{x}$

After putting values of unknown coefficients, the field components for $\mathrm{x}$-directed current are described below:-

$E_{z 1}=\frac{k_{x} k_{2} \cos \left(k_{1} z\right)}{\omega \varepsilon_{0} T_{m}} \tilde{J}_{x}$

$\tilde{H}_{z 1}=\frac{-j k y \sin \left(k_{1} z\right)}{T_{e}} \tilde{J}_{x}$

$\tilde{E}_{z 2}=\frac{k_{1} k x \sin \left(k_{1} h\right)}{j \omega \varepsilon_{0} T_{m}} e^{-j k_{2}(z-h)} \tilde{J}_{x}$

$\tilde{H}_{z 2}=\frac{-j k y \sin \left(k_{1} h\right)}{T_{e}} e^{-j k_{2}(z-h)} \tilde{J}_{x}$

$\tilde{E}_{x}=\frac{-j}{\omega \varepsilon_{0}}\left[\frac{k_{x} k_{1} k_{2} \sin \left(k_{1} h\right)}{\beta^{2} T_{m}}+\frac{k_{y} k_{0}^{2} \sin \left(k_{1} h\right)}{\beta^{2} T_{e}}\right] J_{x}$

$\tilde{E}_{y}=\frac{-j}{\omega \varepsilon_{0}}\left[\frac{k_{y} k_{1} k_{2} \sin \left(k_{1} h\right)}{\beta^{2} T_{m}}-\frac{k_{x} k_{0}^{2} \sin \left(k_{1} h\right)}{\beta^{2} T_{e}}\right] J_{x}$

The electric field expressions can be arranged in matrix form involving spectral domain Green's functions,

$\left[\begin{array}{c}\tilde{E}_{x} \\ \tilde{E}_{y}\end{array}\right]=\left[\begin{array}{ll}\tilde{G}_{x x} & \tilde{G}_{x y} \\ \tilde{G}_{y x} & \tilde{G}_{y y}\end{array}\right]\left[\begin{array}{c}\tilde{J}_{x} \\ \tilde{J}_{y}\end{array}\right]$

\section{RESULTS}

The results have been obtained using MATLAB 2007b. MATLAB allows matrix manipulations, plotting of functions and data implementation of algorithms and creation of user interfaces. Figure.3. shows the few Entire Domain Basis function which are used to obtain accurate results from a method of moment procedure. Because numerical convergence depends strongly on the basis function chosen for the expansion of the patch surface current density. Behavior of surface current density is shown in figure 4 . Which indicate that surface current is maximum at the feed point $\mathrm{x}=\mathrm{y}=0$ and decreases at the outer boundary. Green's function behavior for planar structure as a function of $\mathrm{k}_{\mathrm{x}}$ and $\mathrm{k}_{\mathrm{y}}$ is shown in figure 5 with their absolute, real and imaginary value.

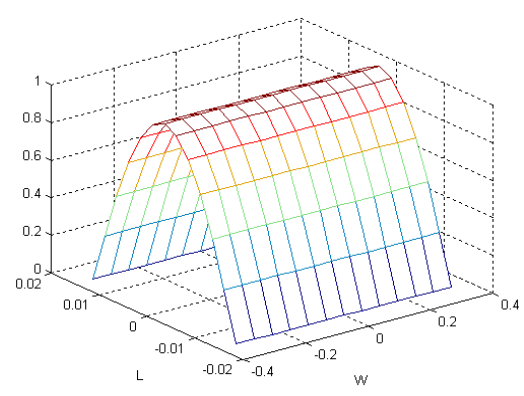

$\mathrm{TM}_{10}$

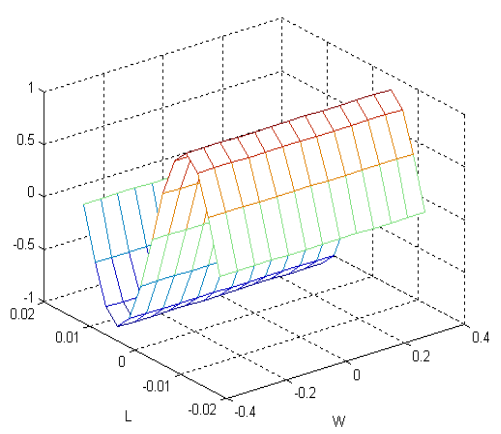

$\mathrm{TM}_{20}$

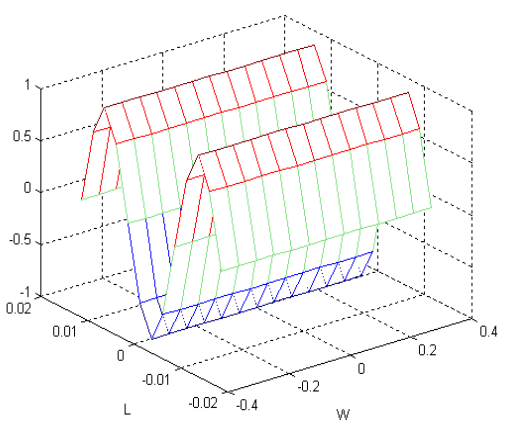

$\mathrm{TM}_{30}$ 


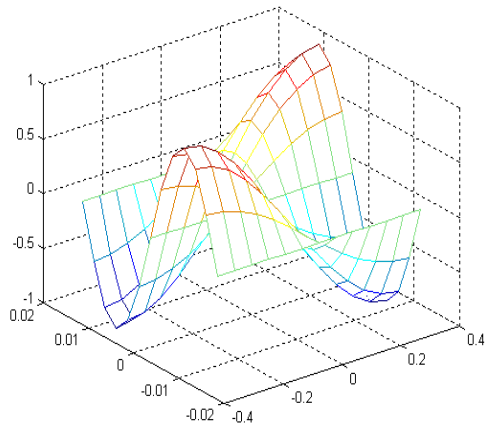

$\mathrm{TM}_{21}$

Fig - 3 Shows the Basis functions Plot for current density in $\mathrm{x}$ direction (without edge singularity)

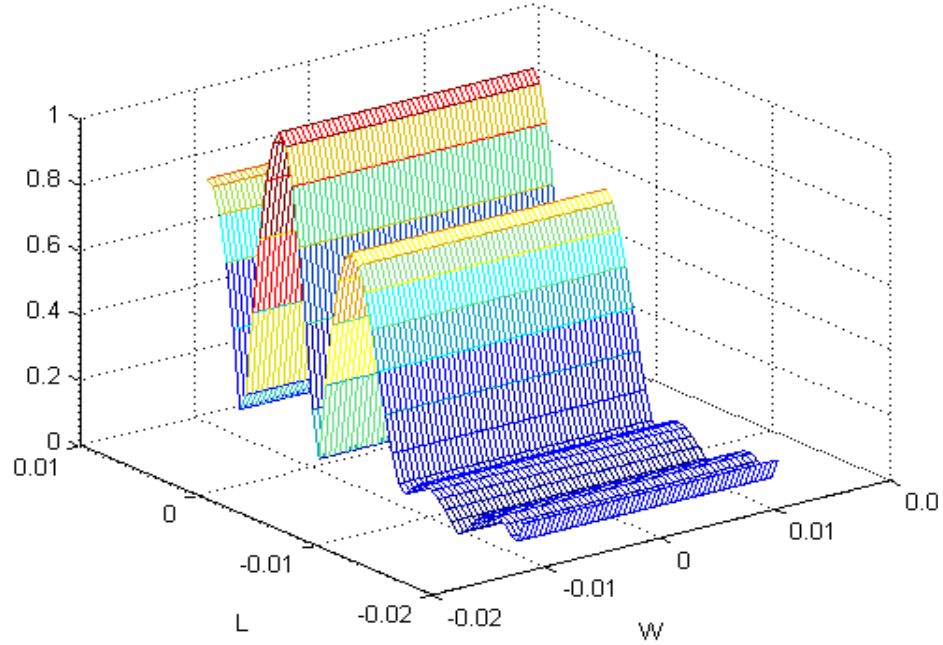

Fig- 4 Shows the surface current density plot for Rectangular patch

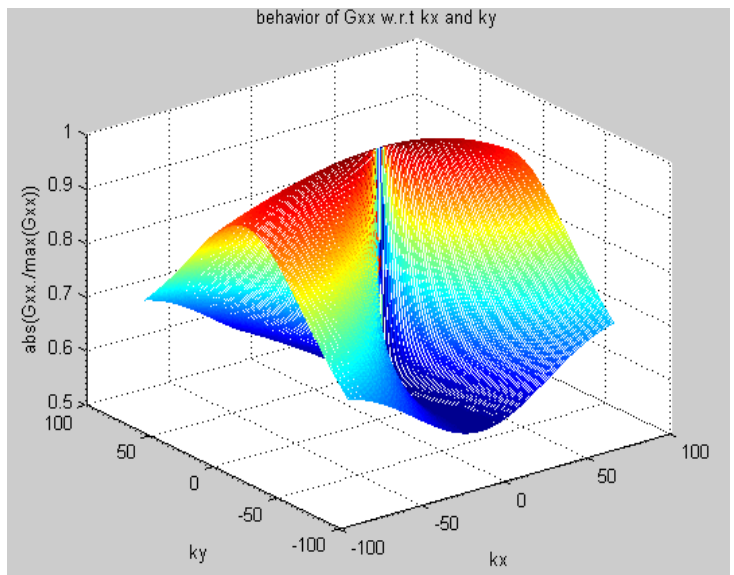

(a)

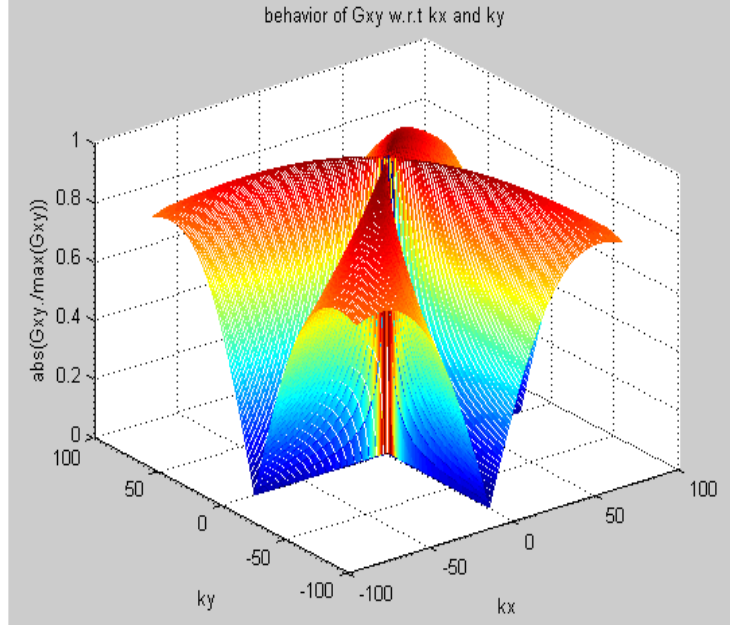

(b)

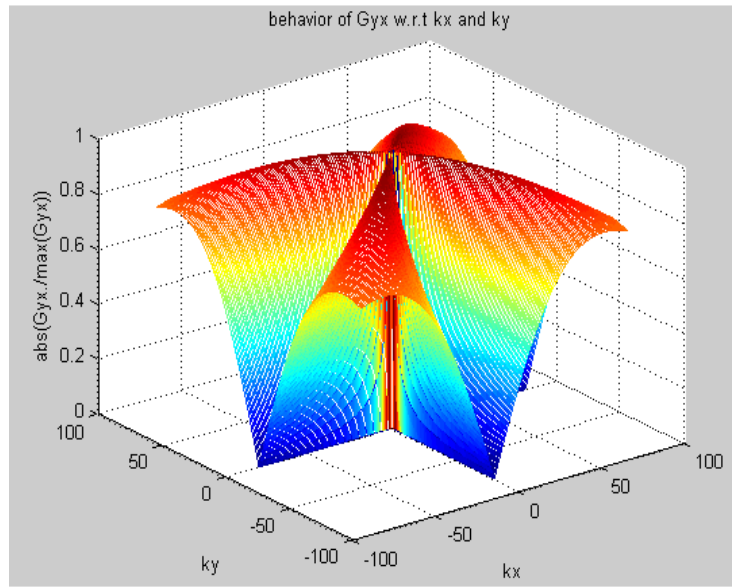

(c)

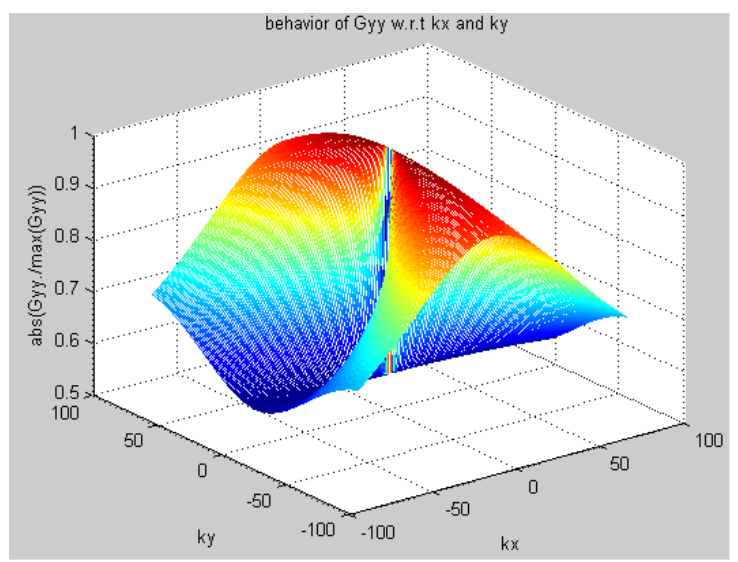

(d)

Fig.5. Behavior of absolute value of Green's Function $G_{x x}, G_{x y}, G_{y x}, G_{y y} w r t k_{x}$ and $k_{y}$. 


\section{CONCLUSIONS}

In this paper we have used the popular and rigorously used spectral domain full wave analysis method in conjunction with method of moment as numerical analysis tool. The electric field due to rectangular patch is obtained by solving integral equation which involves Green's function in spectral domain. The integral equations thus formed are converted into a system of linear equations by the use of method of moment. The Galerkin's procedure with entire domain basis function is used to solve the coupled integral equation. Green's function, surface current density and entire domain basis function for planar structure has been plotted. Simulations are done using MATLAB 2007b.

\section{REFERENCES}

[1] Ramesh Garg, Prakash Bhartia, Inder Bahl, Apisak Ittipiboon, "Microstrip Antenna Design Handbook, Artech House, 2000.

[2] J. R James and P. S Hall, "Handbook of Microstrip Antennas," Peter Peregrinus Ltd., London, United Kingdom 1989.

[3] I. J. Bahl and P. Bhartia, "Microstrip Antennas," Artech House, Dedham, MA, 1980.

[4] Ahmed I. Khalil, Alexander B. Yakovlev, and Michael B. Steer, "Efficient Method-of-Moments Formulation for the Modeling of Planar Conductive Layers in a Shielded Guided-Wave Structure" IEEE Transactions on Microwave Theory and Techniques, vol. 47, no. 9, Sept. 1999

[5] He. Mang and Xu Xiaowen, "Closed Form Solution for Analyzing Cylindrical Rectangular Microstrip Patch

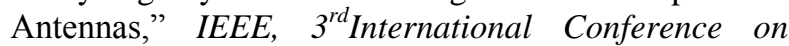
Microwave and Millimeter Wave Tech. Proceedings, 2002.

[6] M. I. Aksun and Gülbin Dural, "Clarification of Issues on the Closed-Form Green's Functions in Stratified Media," IEEE Transactions on Antennas and Propagation, vol. 53, no. 11, November 2005.

[7] Alexander Svezhentsev and Guy Vandenbosch "Model for the Analysis of Microstrip Cylindrical Antennas: Efficient Calculation of the Necessary Green's functions" $11^{\text {th }}$ International Conference on Antennas and Propagation, 17-20 April 2007.

[8] Constantine A. Balanis, “Antenna Theory Analysis and Design," Wiley Interscience, 2005.

[9] S. Karan, V. B. Erturk, and A. Altintas, "Closed-Form Green's Function Representations in Cylindrically Stratified Media for Method of Moments Applications," IEEE Transactions on Antennas and Propagation, Vol. 57, no. 4, April 2009.

[10] Constantine A. Balanis, "Advanced Engineering Electromagnetics", John Willey \& Sons, 1989

[11] Pallavi Singhal, Kuldeep Jaimini, 2013, "Rectangular Microstrip Patch Antenna Design at $3 \mathrm{GHz}$ using Probe Feed" International Journal of Emerging Technology and Advanced Engineering Volume 3, Issue11, November 2013.

\section{APPENDIX}

$$
\begin{aligned}
& T_{m}=k_{2} \cos \left(k_{1} h\right)+j k_{1} \sin \left(k_{1} h\right) \\
& T_{e}=k_{1} \cos \left(k_{1} h\right)+j k_{2} \sin \left(k_{1} h\right) \\
& \tilde{G}_{x x}=\frac{-j}{\omega \varepsilon_{0}}\left[\frac{k_{x}^{2} k_{1} k_{2} \sin \left(k_{1} h\right)}{\beta^{2} T_{m}}+\frac{k_{y}^{2} k_{0}^{2} \sin \left(k_{1} h\right)}{\beta^{2} T_{e}}\right] \\
& \tilde{G}_{x y}=\frac{-j}{\omega \varepsilon_{0}}\left[\frac{k_{x} k_{y} k_{1} k_{2} \sin \left(k_{1} h\right)}{\beta^{2} T_{m}}-\frac{k_{x} k_{y} k_{0}^{2} \sin \left(k_{1} h\right)}{\beta^{2} T_{e}}\right] \\
& \tilde{G}_{y y}=\frac{-j}{\omega \varepsilon_{0}}\left[\frac{k_{y}^{2} k_{1} k_{2} \sin \left(k_{1} h\right)}{\beta^{2} T_{m}}+\frac{k_{x}^{2} k_{0}^{2} \sin \left(k_{1} h\right)}{\beta^{2} T_{e}}\right] \\
& \tilde{G}_{y x}=\tilde{G}_{x y}
\end{aligned}
$$

